\title{
Pervasive Developmental Disorders
}

National Institute of Neurological Disorders and Stroke (NINDS)

\section{Source}

National Institute of Neurological Disorders and Stroke (NINDS). Pervasive

Developmental Disorders Information Page.

The diagnostic category of pervasive developmental disorders (PDD) refers to a group of disorders characterized by delays in the development of socialization and communication skills. Parents may note symptoms as early as infancy, although the typical age of onset is before 3 years of age. Symptoms may include problems with using and understanding language; difficulty relating to people, objects, and events; unusual play with toys and other objects; difficulty with changes in routine or familiar surroundings, and repetitive body movements or behavior patterns. Autism (a developmental brain disorder characterized by impaired social interaction and communication skills, and a limited range of activities and interests) is the most characteristic and best studied PDD. Other types of PDD include Asperger's Syndrome, Childhood Disintegrative Disorder, and Rett's Syndrome. Children with PDD vary widely in abilities, intelligence, and behaviors. Some children do not speak at all, others speak in limited phrases or conversations, and some have relatively normal language development. Repetitive play skills and limited social skills are generally evident. Unusual responses to sensory information, such as loud noises and lights, are also common. 